\title{
Jamming as a Critical Phenomenon: A Field Theory of Zero-Temperature Grain Packings
}

\author{
Silke Henkes and Bulbul Chakraborty \\ Martin Fisher School of Physics, Brandeis University, Mail Stop 057, Waltham, Massachusetts 02454-9110, USA
}

(Received 15 April 2005; published 1 November 2005)

\begin{abstract}
A field theory of frictionless grain packings in two dimensions is shown to exhibit a zero-temperature critical point at a nonzero value of the packing fraction. The zero-temperature constraint of force balance plays a crucial role in determining the nature of the transition. Two order parameters, $\langle z\rangle$, the deviation of the average number of contacts from the isostatic value, and $\langle\phi\rangle$, the average magnitude of the force per contact, characterize the transition from the jammed (high packing fraction) to the unjammed (low packing fraction state). The critical point has a mixed character with the order parameters showing a jump discontinuity but with fluctuations of the contact force diverging. At the critical point, the distribution of $\phi$ shows the characteristic plateau observed in static granular piles. The theory makes falsifiable predictions about the spatial fluctuations of the contact forces.
\end{abstract}

DOI: 10.1103/PhysRevLett.95.198002

PACS numbers: 45.70.- n, 64.70.Pf, 83.80.Fg

Introduction. - In a remarkably diverse range of systems, the transition from a flowing, liquid state to a jammed, solid state is heralded by a dramatic slowing down of relaxations [1]. Does an equilibrium critical point underlie this glassy dynamics? The debate surrounding this question has been spurred by the absence of any obvious static signature accompanying the rapid increase of time scales [1]. Purely dynamical scenarios have been proposed [2] to explain time-scale divergences with no accompanying static divergences. For thermal systems, a different perspective has been offered within the framework of an avoided critical point [3] and a scaling theory based on the existence of a zero-temperature critical point [4]. In a more recent development, it has been suggested that the mechanism of jamming in both thermal and athermal systems is controlled by a zero-temperature critical point ( $J$ point) [5].

Experiments on weakly sheared granular media indicate that at a critical packing fraction there is a transition which is accompanied by slow dynamics, vanishing of mean stress, increasing stress fluctuations, and a change in the distribution of contact forces [6,7]. Simulations indicate a critical point occurring at zero temperature and a packing fraction close to the random close packing value [5]. At this critical point the grain packing is isostatic, having reached the special coordination where all contact forces are completely determined by the packing geometry [1]. A theory based on this observation predicts a diverging length scale associated with the mechanical stability of the network [8]. In a different theoretical approach, an analogy has been drawn between the jamming transition and $k$-core percolation [9].

Collective properties of granular packings. - Granular matter is made up of macroscopic objects for which thermal fluctuations are unimportant. Many different packings of grains can, however, be compatible with a given macroscopic parameter such as the packing fraction and the proper statistical description of such nonthermal assem- blies has been discussed extensively in the literature [1]. A widely used prescription is the microcanonical postulate leading to the Edwards ensemble [10]. The principle of maximum entropy [11] generates the usual rules of equilibrium (thermal) statistical mechanics without appealing to any physical principles. The canonical Boltzmann distribution can, for example, be obtained by maximizing the entropy subject to the constraint of a given, measured, average energy [12]. This approach to statistical mechanics is not restricted to systems in thermal equilibrium and we adopt it to define the statistical weight of grain packings.

The statistical ensemble of grains is constrained by the requirements of mechanical equilibrium. In two dimensions, the force-balance constraint can be rigorously enforced through the mapping of the contact forces to a height field [13] defined on the voids surrounded by grains (cf. Fig. 1). Expressing the microscopic stress tensor in terms of these height fields, we construct a coarse-grained formalism for describing the long-wavelength properties of grain packings.

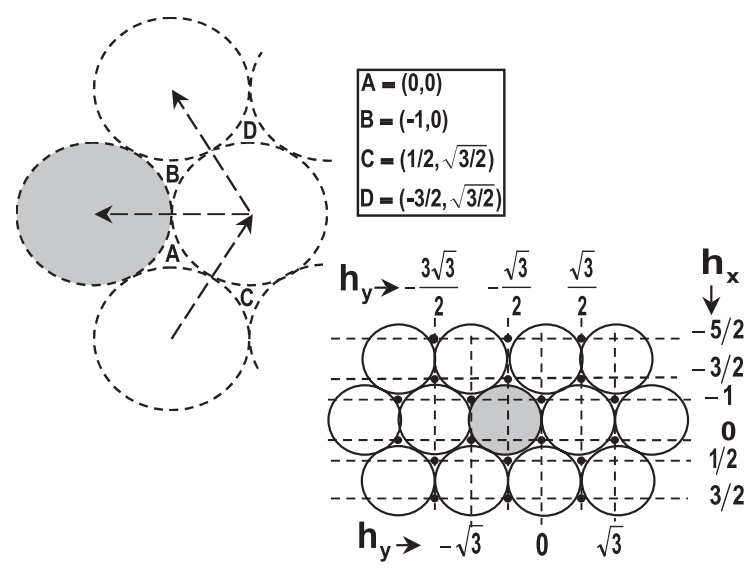

FIG. 1. Top panel: heights $\left(h_{x}, h_{y}\right)$ on voids surrounding a grain of an ordered hexagonal packing with $F=1$. The vectors denote contact forces. Bottom panel: corresponding height contours. 
The statistical field theory based on the height mapping embodies a competition between the compressive effect of increasing packing fractions and an entropic preference for flat height configurations [14] which correspond to force networks that are fragmented at all scales. As shown in this work, this competition leads to the existence of a critical packing fraction separating a disordered phase from an "ordered" one characterized by two order parameters: (i) the magnitude of the force per contact, $\langle\phi\rangle$, and (ii) $\langle z\rangle$, the deviation of the contact number per grain from its isostatic value. At the critical point, the fluctuations around $\langle\phi\rangle$ diverge.

Construction of field theory. - The algorithm of maximum entropy $[11,12]$ specifies that the probability $P\left[\left\{\mathbf{r}_{i}\right\}\right]$ of occurrence of a configuration with grain positions $\left\{\mathbf{r}_{i}\right\}$ be determined by maximizing the entropy, $S[P]=$ $-\sum_{\left\{\mathbf{r}_{i}\right\}} P\left[\left\{\mathbf{r}_{i}\right\}\right] \ln P\left[\left\{\mathbf{r}_{i}\right\}\right]$ subject to appropriate constraints. For $N$ grains in a volume $V$, interacting via short-range repulsive potentials, the constraint is that of fixed average "pressure" [15]: $\sum_{\left\{\mathbf{r}_{i}\right\}} P\left[\left\{\mathbf{r}_{i}\right\}\right] p\left(\left\{\mathbf{r}_{i}\right\}\right)=\sum_{\left\{\mathbf{r}_{i}\right\}} P\left[\left\{\mathbf{r}_{i}\right\}\right] \times$ $\left.\sum_{i j} r_{i j} \frac{\partial U}{\partial r}\right|_{r_{i j}}=\langle p\rangle$ (U is the interaction potential) and the resulting distribution is:

$$
P\left[\left\{\mathbf{r}_{i}\right\}\right]=(1 / Z) \exp \left[\alpha p\left(\left\{\mathbf{r}_{i}\right\}\right)\right] .
$$

The partition function, $Z(\alpha)=\sum_{\left\{\mathbf{r}_{i}\right\}}^{\prime} P\left[\left\{\mathbf{r}_{i}\right\}\right]$, is the generating function of all statistical averages. The Lagrange multiplier $\alpha$ plays the role of inverse temperature: $\alpha=-\partial S / \partial\langle p\rangle$ and the prime on the summation restricts it to grain configurations satisfying the equations of mechanical equilibrium for frictionless packings in $d$ dimensions:

$$
\begin{gathered}
d N \text { equations }: \sum_{j} F_{i j} \frac{\mathbf{r}_{i j}}{\left|\mathbf{r}_{i j}\right|}=0 \\
\langle z\rangle N / 2 \text { equations }: F_{i j}=f\left(\mathbf{r}_{i j}\right) .
\end{gathered}
$$

Here $\langle z\rangle$ is the average number of contacts per grain, $F_{i j}$ is the magnitude of the contact force between grains $i$ and $j$, and $f\left(\mathbf{r}_{i j}\right)$ specifies the force law. At the isostatic point, $\langle z\rangle=z_{\text {iso }}=2 d$, the number of equations in Eq. (2) is exactly equal to the number of unknowns $[16,17]$ and, therefore, the forces are uniquely determined. For $\langle z\rangle>$ $z_{\text {iso }}$, Eqs. (2) and (3) are coupled with a strength $\epsilon$ which depends on the compressivity of the grains: [17]:

$$
\epsilon=\frac{\langle F\rangle}{\left\langle r_{i j}\right\rangle}\left\langle\frac{d F_{i j}}{d r_{i j}}\right\rangle^{-1}
$$

For hard spheres, $\epsilon=0$, the two equations are decoupled and the only packings for which $\left\{F_{i j}\right\}$ and $\left\{\mathbf{r}_{i j}\right\}$ can be determined are the isostatic ones [16]. For $\epsilon \ll 1$, small variations in positions can lead to significant force changes. In this weak-coupling limit, the partition function can be calculated by summing over all the solutions, $\left\{F_{i j}\right\}$, to Eq. (2) for a given set $\left\{\mathbf{r}_{i}\right\}$ and imposing a Gaussian constraint:

$$
Z(\alpha)=\sum_{\left\{\mathbf{r}_{i}\right\},\left\{F_{i j}\right\}} e^{\left(\alpha \sum_{i} p_{i}\right)} e^{-\epsilon / 2 \sum_{i}\left(p_{i}-p_{i}^{0}\right)^{2}} .
$$

Here $p_{i}=\sum_{j} r_{i j} f\left(r_{i j}\right)$ is the pressure on grain $i$ calculated from $\left\{\mathbf{r}_{i}\right\}$ and $p_{i}^{0}=\sum_{j} r_{i j} F_{i j}$ is calculated from $\left\{F_{i j}\right\}$.

Height map. - The sum over $F_{i j}$ is only over the force configurations which satisfy force balance and, therefore, can be mapped to the height field. The mapping rule is that the height field gets incremented by the contact force crossed while traveling around a grain in a counterclockwise direction: $\mathbf{F}_{i j}=\mathbf{h}_{j^{\prime}}-\mathbf{h}_{i^{\prime}}=-\mathbf{F}_{j i}$ where $j^{\prime}$ and $i^{\prime}$ are the voids bracketing the contact $i j$ [13] (cf. Fig. 1). Since $\sum_{j} \mathbf{F}_{i j}=\mathbf{0}$, the mapping of forces to heights is one to one, up to an arbitrary choice of a single height. The coarse graining of these height fields has been discussed in detail in Ref. [13]. For frictionless grains, $\mathbf{F}_{i j} \| \mathbf{r}_{i j}$, and upon coarse graining the heights over a mesoscopic region with a length scale much larger than the grain radius but smaller than a typical length scale over which the height fields vary [13], this constraint translates to $\boldsymbol{\nabla} \cdot \mathbf{h}=0$ [18]. The divergenceless height field can be expressed in terms of a scalar potential $\psi: h_{x}=\partial_{y} \psi ; h_{y}=-\partial_{x} \psi$. It has been shown in Ref. [13] that the microscopic stress tensor, coarse grained over a mesoscopic region of area $A$, around the point $\mathbf{r}: \hat{\sigma}^{0}(\mathbf{r})=(1 / A) \sum_{j \subset A} \sum_{k} \mathbf{r}_{j k} \mathbf{F}_{j k}$, is:

$$
\hat{\sigma}^{0}=\left[\begin{array}{cc}
\partial_{y} h_{x} & \partial_{y} h_{y} \\
-\partial_{x} h_{x} & -\partial_{x} h_{y}
\end{array}\right]=\left[\begin{array}{cc}
\partial_{y}^{2} \psi & -\partial_{x} \partial_{y} \psi \\
-\partial_{x} \partial_{y} \psi & \partial_{x}^{2} \psi
\end{array}\right],
$$

where the second equality is special to frictionless grains. Since the coarse-grained pressure is $p^{0}(\mathbf{r}) \equiv \operatorname{Tr} \hat{\sigma}=$ $\nabla^{2} \psi(\mathbf{r})$, the partition function, Eq. (5), can be written in terms of the unconstrained field $\psi$.

Coarse graining the heights leads to a weight $\Omega[\psi]$ which counts the number of microscopic $\left\{F_{i j}\right\}$ configurations giving rise to the same $\psi$ field. Arguments similar to the ones employed in height maps of loop models [14] lead to $\Omega[\psi] \sim e^{-(1 / 2) \int d^{d} r\left(\left|\nabla h_{x}\right|^{2}+\left|\nabla h_{y}\right|^{2}\right)}=e^{-(1 / 2) \int d^{d} r\left(\nabla^{2} \psi\right)^{2}}$ since flat interfaces have many equal height contours and the forces within the regions bounded by these contours can be independently rearranged without affecting force balance. The forces in the ordered configuration of Fig. 1, with completely tilted interfaces (in $h_{x}$ and $h_{y}$ ), cannot be rearranged locally.

At the isostatic point, $z=z_{\text {iso }}$, there is a unique solution to Eq. (2) and $p=\nabla^{2} \psi$. Expanding the pressure around this point:

$$
\begin{aligned}
p(\mathbf{r}) & =\nabla^{2} \psi+\left.\left(z-z_{\text {iso }}\right) \frac{\Delta p}{\Delta z}\right|_{z_{\text {iso }}} \\
& =\nabla^{2} \psi+\left(z-z_{\text {iso }}\right) \frac{\nabla^{2} \psi}{z_{\text {iso }}}=z \frac{\nabla^{2} \psi}{z_{\text {iso }}} .
\end{aligned}
$$

The second set of equations follow by noting that the extra pressure, $\left.\frac{\Delta p}{\Delta z}\right|_{z_{\text {iso }}}$, due to the introduction of an additional 
contact at the isostatic point, is $\frac{\nabla^{2} \psi}{z_{\text {iso }}}$. As the mapping from $F_{i j} \rightarrow \psi$, the mapping from $\mathbf{r}_{i}$ to the coarse-grained pressure, $p(\mathbf{r})$, leads to a weight $\omega[p] \sim \exp \left[-K|(\nabla p)|^{2}\right]$. This follows from the observation that, entropically, it is favorable to have the grains occupy as much of the volume as possible, leading to small spatial variations of the packing density and $p(\mathbf{r})$.

With these weights, the partition function is:

$$
\begin{aligned}
Z= & \sum_{\{z\},\{\psi\}} \Omega[\psi] \omega[p]\left\{e^{\alpha \int d^{d} r p(r)}\right\}\left\{e^{-(\epsilon / 2) \int d^{d} r\left[p(r)-p^{0}(r)\right]^{2}}\right\} \\
= & \sum_{\{z\},\{\psi\}} e^{-H[\psi, z]} \\
H= & \sum_{\mathbf{q}}\left[\frac{1}{2}\left|\phi_{\mathbf{q}}\right|^{2}-\alpha\left(z_{\text {iso }} \delta_{\mathbf{q}}+z_{-\mathbf{q}}\right) \phi_{\mathbf{q}}\right] \\
& +V^{-1} \sum_{\mathbf{q}_{1}, \mathbf{q}_{2}, \mathbf{q}_{3}, \mathbf{q}_{4}}\left[\frac{\epsilon}{2}-\frac{K}{2}\left(\mathbf{q}_{1}+\mathbf{q}_{3}\right) \cdot\left(\mathbf{q}_{2}+\mathbf{q}_{4}\right)\right] \\
& \times\left[z_{\mathbf{q}_{1}} z_{\mathbf{q}_{2}} \boldsymbol{\phi}_{\mathbf{q}_{3}} \phi_{\mathbf{q}_{4}} \delta_{\left.\mathbf{q}_{1}+\mathbf{q}_{2}+\mathbf{q}_{3}+\mathbf{q}_{4}\right]}\right]
\end{aligned}
$$

Here, $\phi=\nabla^{2} \psi$ is the pressure per contact [cf. Eq. (6)], and the field $z$ has been redefined to $z-z_{\text {iso }}$ which is restricted to the set of integers. The parameters $\alpha, \epsilon$, and $K$ have been scaled to absorb resulting factors of $z_{\text {iso }}$.

Critical point. - The Hamiltonian $H$ reflects the competition between $\Omega[\psi]$ favoring $\phi=0$ and the "field" $\alpha$ favoring nonzero $\phi$. It provides a model for studying the response functions of grain packings with small but finite $\epsilon$. We assume that the integer restriction on $z$ can be ignored [19] as long as $\epsilon \neq 0$.

In investigating whether or not there is a finite- $\alpha$ phase transition involving the vanishing of one or more order parameters, the fields in the Hamiltonian in Eq. (7) are expanded around their average values; $\phi_{\mathbf{q}}=\left\langle\phi_{\mathbf{q}}\right\rangle+\zeta_{\mathbf{q}}, z_{\mathbf{q}}=$ $\left\langle z_{\mathbf{q}}\right\rangle+\eta_{\mathbf{q}}$ with $H=H_{0}\left(\left\langle\phi_{q}\right\rangle,\left\langle z_{q}\right\rangle\right)+H_{1}\left(\left\langle\phi_{q}\right\rangle,\left\langle z_{q}\right\rangle ; \zeta_{q}, \eta_{q}\right)$. The order parameters $\left\langle\phi_{q}\right\rangle$ and $\left\langle z_{q}\right\rangle$ are obtained by minimizing the effective potential (a Landau-Ginzburg type free energy functional), $\Gamma\left(\left\langle\phi_{q}\right\rangle,\left\langle z_{q}\right\rangle\right)=H_{0}\left(\left\langle\phi_{q}\right\rangle,\left\langle z_{q}\right\rangle\right)-$ $\ln \left(\int \Pi_{\mathbf{q}} d \zeta_{\mathbf{q}} \Pi_{\mathbf{q}} d \eta_{\mathbf{q}} e^{-H_{1}}\right)$ [20]. The simplest nontrivial approximation, is obtained by calculating the fluctuations, $\left\langle\left|\zeta_{\mathbf{q}}\right|^{2}\right\rangle$ and $\left\langle\left|\eta_{\mathbf{q}}\right|^{2}\right\rangle$, at the loop level, replacing all 4-point averages by 2-point averages [20], and assuming spatially uniform order parameters: $\langle\phi\rangle \equiv\left\langle\phi_{q=0}\right\rangle,\langle z\rangle \equiv\left\langle z_{q=0}\right\rangle$. To leading order in $\epsilon$ :

$$
\begin{aligned}
\left\langle\left|\zeta_{\mathbf{q}}\right|^{2}\right\rangle^{-1} & =1+\epsilon\langle z\rangle^{2}-1 /\langle\phi\rangle^{2}+K\langle z\rangle^{2} q^{2} \\
\left\langle\left|\eta_{\mathbf{q}}\right|^{2}\right\rangle^{-1} & =\left(\epsilon+K q^{2}\right)\langle\phi\rangle^{2} .
\end{aligned}
$$

Minimizing $\Gamma(\langle\phi\rangle,\langle z\rangle)$ gives:

$$
\begin{aligned}
\langle\phi\rangle-\alpha\left(z_{\text {iso }}+\langle z\rangle\right)+\epsilon\langle z\rangle^{2}\langle\phi\rangle+\frac{1}{\langle\phi\rangle} & =0 \\
\epsilon\left(\langle\phi\rangle^{2}+\frac{1}{1-1 /\langle\phi\rangle^{2}}\right)\langle z\rangle-\alpha\langle\phi\rangle & =0,
\end{aligned}
$$

and the solutions near $\alpha_{c}=2 / z_{\text {iso }}$ are:

$$
\begin{aligned}
\langle\phi\rangle & =\left(\alpha / \alpha_{c}\right)\left[1+\left(1-\alpha_{c} / \alpha\right)^{1 / 2}\right] \\
\langle z\rangle & =(\alpha / \epsilon)\left(1-\alpha_{c} / \alpha\right)^{1 / 2} .
\end{aligned}
$$

For $\alpha \geq \alpha_{c}$, there is an ordered phase characterized by two order parameters. For $\alpha<\alpha_{c}, \Gamma(\langle\phi\rangle,\langle z\rangle)$ ceases to have any local minima or maxima (cf. Fig. 2) and $\langle\phi\rangle$ jumps discontinuosly to 0 : the physical limit of its allowed values. From Eqs. (8) and (10), as $\alpha \rightarrow \alpha_{c}, \phi \rightarrow 1$, and the $q=0$ force fluctuations diverge: $\left\langle\phi^{2}\right\rangle-\langle\phi\rangle^{2} \sim \frac{1}{1-1 /\langle\phi\rangle^{2}} \sim(1-$ $\left.\alpha_{c} / \alpha\right)^{-1 / 2}$. This type of transition is indicative of the end of a line of metastable equilibrium similar to spinodal critical points. Unlike spinodals, however, the transition is accompanied by the disappearance of any local minimum: a phenomenon observed in models with rigid constraints such as certain dimer models [21].

Structure factor and correlation length. - Since the critical point is marked by an instability in $\phi=\nabla^{2} \psi$, it is necessary to include the next higher order derivative term, $|\nabla \phi|^{2}$, and the fluctuations [cf. Eq. (8)] acquire new terms proportional to $q^{2}$. The structure factor of the magnitude of the contact forces (the $\phi$ field), $S(|\mathbf{q}|) \equiv\left\langle\left|\zeta_{\mathbf{q}}\right|^{2}\right\rangle$, describes the spatial fluctuations:

$$
\begin{aligned}
S(q) & \equiv\left\langle\left|\zeta_{q}\right|^{2}\right\rangle \\
& =\frac{1}{1-1 /\langle\phi\rangle^{2}+\left(\epsilon+K q^{2}\right)\langle z\rangle^{2}+K_{\phi} q^{2}} \\
& =\frac{\xi^{2}}{1+\left(\alpha^{2} / \epsilon^{2}\right)\left(\epsilon+K q^{2}\right) \xi^{-2}+K_{\phi} q^{2} \xi^{2}},
\end{aligned}
$$

where $K_{\phi}$ is the coefficient of the $|\nabla \phi|^{2}$ term, and the correlation length $\xi=\sqrt{\frac{1}{1-1 /\langle\phi\rangle^{2}}}$ which diverges as $(1-$ $\left.\alpha_{c} / \alpha\right)^{-1 / 4}$. The appearance of the diverging length scales and the specific form of $S(q)$ are predictions of the theory that should be testable in experiments and simulations. A correlation length exponent of $1 / 4$ has been discussed in theories and simulations of the $J$ point $[5,8,9]$.

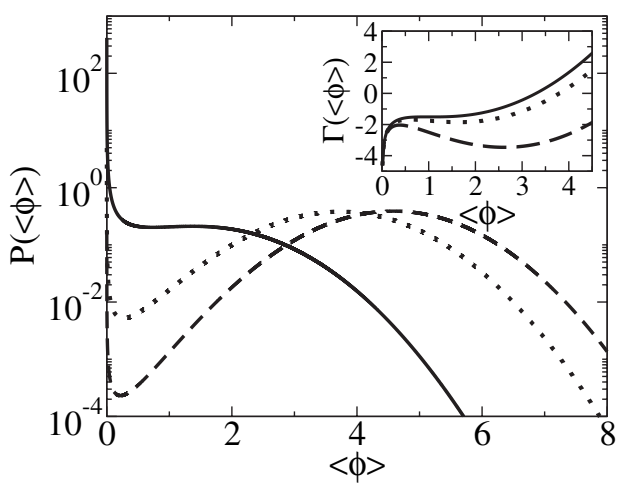

FIG. 2. The distribution $P(\langle\phi\rangle)$ of contact forces (from right to left) $\alpha-\alpha_{c}=0.7,0.5$, and 0.025 . The inset shows the $\langle z\rangle=0$ cut of the potential, $\Gamma(\langle\phi\rangle,\langle z\rangle)$, at different $\alpha$ with $\alpha \rightarrow \alpha_{c}$ from bottom to top. 
Distribution of contact forces. - Experiments [6,7] and simulations [5,17] have shown that changes in the force distribution $P(F)$ are associated with transitions involving the vanishing of stress. In the field theory, $\phi$ corresponds to $F$, modulo a microscopic length scale [18] and the distribution (configuration to configuration variation) of $\langle F\rangle$, the spatial average of the force (magnitude) is $P(\langle F\rangle) \equiv$ $P(\langle\phi\rangle)$. The latter is determined by the free energy functional $\Gamma: P(\langle\phi\rangle) \simeq e^{-\Gamma(\langle\phi\rangle,\langle z(\langle\phi\rangle)\rangle)}$. In the critical regime where $\langle z(\langle\phi\rangle)\rangle \simeq 0, \quad \Gamma(\langle\phi\rangle) \simeq(1 / 2)\langle\phi\rangle^{2}-\alpha z_{\text {iso }}\langle\phi\rangle+$ $\ln (\langle\phi\rangle)$. The $\ln (\langle\phi\rangle)$ term results from integrating out the $z$ field and embodies the physical effect of large contact number fluctuations for small contact forces. As seen in Fig. 2, the peak in $P(\langle\phi\rangle)$ gives away to a plateau as $\alpha \rightarrow$ $\alpha_{c}$. The plateau signals diverging force fluctuations and vanishing shear modulus $\sim 1 /\left(\left\langle\phi^{2}\right\rangle-\langle\phi\rangle^{2}\right)$. The distribution of $\langle\phi\rangle$ does not exhibit the exponential tail characteristic of the distribution of the individual forces. This feature reflects a stress redistribution at the particle level and disappears upon any spatial averaging [6,22]. The diverging width of $P(\langle F\rangle)$ was inferred in the simulations [5] through an observed lack of self-averaging.

Conclusions. - We have demonstrated the existence of a zero-temperature critical point separating a jammed phase with finite yield stress from an unjammed phase. The critical point is characterized by a diverging correlation length associated with force fluctuations and marked changes in the shape of the force distribution. In experiments where the grain packing is under a fixed loading, the system will generically be in a two-phase coexistence regime since the force (or pressure) is the order parameter of the transition. In these situations, identifying the critical point will require a better understanding of the nature of the phase coexistence.

The force-balance constraint is violated by any form of driving and the stability of the critical point to weak driving needs to be analyzed to determine the dynamical behavior of grain packings. If the critical point is stable then glassy dynamics follows from general scaling arguments [4,23]. The extension of the theory to frictional packings is within reach since the loop-force formalism exists [13]. Although the similarities between two and three dimensions, observed in simulations, suggest that an extension to higher dimensions is possible, this remains an open question.

The authors would like to thank Jané Kondev for many insightful comments including the suggestion about the entropy associated with a coarse-grained pressure field. B. C. would like to acknowledge useful discussions with Tom Witten, Sid Nagel, Andrea Liu, Leo Silbert, Matthew Wyart, and Ilya Gruzberg and the hospitality of the James Franck Institute. This work was supported by the NSF Grants No. DMR-0207106 and No. DMR-0403997.
[1] See, e.g., recent reviews in J.-P. Bouchaud, Slow Relaxations and Nonequilibrium Dynamics in Condensed Matter, edited by J.-L. Barrat, J. Dalibard, M. Feigelman, and J. Kurchan (Springer, Berlin, 2003) and articles in Jamming and Rheology, edited by A. J. Liu and S. Nagel (Taylor \& Francis, London, 2001).

[2] C. Toninelli, G. Biroli, and D. S. Fisher, Phys. Rev. Lett. 92, 185504 (2004); J. P. Garrahan and D. Chandler, Phys. Rev. Lett. 89, 035704 (2002); R. Stinchcombe and M. Depken, Phys. Rev. Lett. 88, 125701 (2002).

[3] G. Tarjus and D. Kivelson, in Jamming and Rheology, edited by A. Lui and S.R. Nagel (Taylor \& Francis, New York, 2001).

[4] J. P. Sethna, J. D. Shore, and M. Huang, Phys. Rev. B 47, 14661 (1993).

[5] C.S. O'Hern, L.E. Silbert, A. J. Liu, and S. R. Nagel, Phys. Rev. E 68, 011306 (2003); C. S. OHern, S. A. Langer, A. J. Liu, and S. R. Nagel, Phys. Rev. Lett. 88, 075507 (2002); L. E. Silbert, A. J. Liu, and S. R. Nagel, Phys. Rev. Lett. 95, 098301 (2005).

[6] D. Howell, R. P. Behringer, and C. Veje, Phys. Rev. Lett. 82, 5241 (1999); C. Veje, D. Howell, and R. P. Behringer, Phys. Rev. E 59, 739 (1999).

[7] D. M. Mueth, H. M. Jaeger, and S. R. Nagel, Phys. Rev. E 57, 3164 (1998); E. I. Corwin, H. R. Jaeger, and S. R. Nagel (to be published).

[8] M. Wyart, S.R. Nagel, and T.A. Witten, cond-mat/ 0409687.

[9] J. Schwarz, A.J. Liu, and L. Q. Chayes, cond-mat/ 0410595.

[10] S. F. Edwards and C. C. Mounfield, Physica (Amsterdam) 226A, 1 (1996).

[11] E. T. Jaynes, Phys. Rev. 106, 620 (1957).

[12] D. Chandler, Introduction to Modern Statistical Mechanics (Oxford University Press, New York, 1987).

[13] Robin C. Ball and Raphael Blumenfeld, Phys. Rev. Lett. 88, 115505 (2002).

[14] J. Kondev and C. L. Henley, Nucl. Phys. B464, 540 (1996), and references therein.

[15] As defined, the pressure is actually an extensive quantity equal to the pressure times volume but we refer to it as pressure for simplicity.

[16] A. V. Tkachenko and T. A. Witten, Phys. Rev. E 60, 687 (1999).

[17] J.H. Snoeijer, T. J.H. Vlugt, M. van Hecke, and W. van Saarloos, Phys. Rev. Lett. 91, 072303 (2003).

[18] S. Henkes and Bulbul Chakraborty (to be published).

[19] S. T. Chui and J. D. Weeks, Phys. Rev. B 14, 4978 (1976).

[20] D. J. Amit, Field Theory, the Renormalization Group, and Critical Phenomena (World Scientific, Singapore, 1984), Chap. 5-6.

[21] H. Yin and B. Chakraborty, Phys. Rev. E 65, 036119 (2002).

[22] S. N. Coppersmith, C.-h. Liu, S. Majumdar, O. Narayan, and T. A. Witten, Phys. Rev. E 53, 4673 (1996).

[23] Satya N. Majumdar, Dibyendu Das, Jane' Kondev, and Bulbul Chakraborty, Phys. Rev. E 70, 060501(R) (2004). 